\title{
A Study on Mastectomy Samples to Evaluate Breast Imaging Quality and Potential Clinical Relevance of Differential Phase Contrast Mammography
}

Hauser, Nik ; Wang, Zhentian ; Kubik-Huch, Rahel A ; Trippel, Mafalda ; Singer, Gad ; Hohl, Michael K ; Roessl, Ewald ; Köhler, Thomas ; van Stevendaal, Udo ; Wieberneit, Nataly ; Stampanoni, Marco

DOI: https://doi.org/10.1097/RLI.0000000000000001

Posted at the Zurich Open Repository and Archive, University of Zurich ZORA URL: https://doi.org/10.5167/uzh-107076

Journal Article

Published Version

Originally published at:

Hauser, Nik; Wang, Zhentian; Kubik-Huch, Rahel A; Trippel, Mafalda; Singer, Gad; Hohl, Michael K; Roessl, Ewald; Köhler, Thomas; van Stevendaal, Udo; Wieberneit, Nataly; Stampanoni, Marco (2014). A Study on Mastectomy Samples to Evaluate Breast Imaging Quality and Potential Clinical Relevance of Differential Phase Contrast Mammography. Investigative Radiology, 49(3):131-137.

DOI: https://doi.org/10.1097/RLI.0000000000000001 


\title{
A Study on Mastectomy Samples to Evaluate Breast Imaging Quality and Potential Clinical Relevance of Differential Phase Contrast Mammography
}

\author{
Nik Hauser, PhD, * Zhentian Wang, PhD, † Rahel A. Kubik-Huch, MD, MPH, \\ Mafalda Trippel, MD, \& Gad Singer, MD, § Michael K. Hohl, MD, * \\ Ewald Roessl, PhD,// Thomas Köhler, PhD,// Udo van Stevendaal, PhD,// \\ Nataly Wieberneit, PhD, I and Marco Stampanoni, PhD†\#
}

\begin{abstract}
Objectives: Differential phase contrast and scattering-based x-ray mammography has the potential to provide additional and complementary clinically relevant information compared with absorption-based mammography. The purpose of our study was to provide a first statistical evaluation of the imaging capabilities of the new technique compared with digital absorption mammography. Materials and Methods: We investigated non-fixed mastectomy samples of 33 patients with invasive breast cancer, using grating-based differential phase contrast mammography (mammoDPC) with a conventional, low-brilliance x-ray tube. We simultaneously recorded absorption, differential phase contrast, and smallangle scattering signals that were combined into novel high-frequency-enhanced images with a dedicated image fusion algorithm. Six international, expert breast radiologists evaluated clinical digital and experimental mammograms in a 2-part blinded, prospective independent reader study. The results were statistically analyzed in terms of image quality and clinical relevance.

Results: The results of the comparison of mammoDPC with clinical digital mammography revealed the general quality of the images to be significantly superior $(P<0.001)$; sharpness, lesion delineation, as well as the general visibility of calcifications to be significantly more assessable $(P<0.001)$; and delineation of anatomic components of the specimens (surface structures) to be significantly sharper $(P<0.001)$. Spiculations were significantly better identified, and the overall clinically relevant information provided by mammoDPC was judged to be superior $(P<0.001)$.
\end{abstract}

Conclusions: Our results demonstrate that complementary information provided by phase and scattering enhanced mammograms obtained with the

Received for publication May 30, 2013; and accepted for publication, after revision, August 27, 2013.

From the *Department of Gynecology and Obstetrics, Interdisciplinary Breast Center Baden, Kantonsspital Baden, Baden; †Swiss Light Source, Paul Scherrer Institute, Villigen; †Department of Radiology, §Institute of Pathology, Kantonsspital Baden, Baden, Switzerland; ||Philips Research Laboratories, Innovative Technologies; TPhilips Medical Systems DMC GmbH, Hamburg, Germany; and \#Institute for Biomedical Engineering, University and Eidgenössische Technische Hochschule Zürich, Zürich, Switzerland.

Conflict of interest and sources of funding: E.R., T.K., and U.S. are employees of Philips Research Laboratories, Innovative Technologies, Hamburg, Germany. N.W. is an employee of Philips Medical Systems DMC GmbH, Hamburg, Germany.

N.H. and M.S. conceived the study. N.H. selected the patients, requested written informed consent of all patients as requested by the ethical committee, and performed the surgical interventions at the Kantonsspital Baden, Baden, Switzerland. M.T. prepared and, together with G.S., finalized the histopathologic analysis of the tissue samples at Kantonsspital Baden. R.K. critically revised the article after the blinded reader study and statistical analysis were completed. Z.W. and M.S. designed and set up the differential phase contrast mammography setup at the Paul Scherrer Institute. Z.W. and M.S. performed the phase contrast mammography measurement at the Paul Scherrer Institute. N.W. and N.H. instructed the readers. N.H., Z.W., R.K., M.H., E.R., T.K., U.S., N.W., and M.S. analyzed the data. N.H. and M.S. wrote the article. All the coauthors discussed the results and contributed to the final version of the article.

Reprints: Nik Hauser, PhD, Frauenklinik, Interdisziplinäres Brustzentrum Baden, Kantonsspital Baden, CH-5404 Baden, Switzerland. E-mail: nik.hauser@ksb.ch; Marco Stampanoni, PhD, Swiss Light Source, Paul Scherrer Institute, WBBA/216, CH-5232 Villigen, Switzerland. E-mail: marco.stampanoni@psi.ch.

Copyright (C) 2014 by Lippincott Williams \& Wilkins

ISSN: 0020-9996/14/4903-0131
mammoDPC approach deliver images of generally superior quality. This technique has the potential to improve radiological breast diagnostics.

Key Words: differential phase contrast imaging, mammography, breast imaging, grating interferometry, reader study

(Invest Radiol 2014;49: 131-137)

$P_{h e r}^{h}$ hase contrast and scattering-based (dark-field) x-ray imaging has been shown to be potentially revolutionizing radiological approaches to breast imaging because they are intrinsically capable of detecting subtle differences in the electron density of the tissue (phase signal) and of measuring the amount of small-angle scattering induced by microscopic density fluctuations in the specimen (scattering signal). ${ }^{1-5}$ Fully digital absorption-based mammography, the current clinical standard for screening and diagnosis, ${ }^{6,7}$ is an imperfect modality and has some limitations in sensitivity and falsepositive rates, particularly in women with generally dense breast tissue and fibroglandular tissue as well as heterogeneously dense and extremely dense breasts. ${ }^{7-10}$ Therefore, mammography could experience significant gains (ie, soft-tissue contrast enhancement) from $\mathrm{x}$-ray phase contrast imaging as a highly sensitive, possibly cheap, and fast method, compared with existing approaches, for example, MRI ${ }^{11}$ or tomosynthesis. ${ }^{12,13}$ In a preliminary work, we used grating interferometry to simultaneously record the differential phase and smallangle scattering signals of native, whole-breast samples, in addition to the conventional absorption signal. ${ }^{14}$ We showed that the complementary information that was obtained may indeed allow improved diagnostic capabilities. In this work, we tested the hypothesis that phase contrast and dark-field signals increase the sharpness and delineation of lesions within breast tissue and may lead to a generally increased quality of radiological images. For this purpose, we designed an international, blinded reader study involving experienced breast radiologists evaluating several diagnostic aspects on mastectomy images obtained with both absorption-based clinical digital mammography and our novel phase contrast-enhanced technique.

Published results so far reported the use of synchrotron radiation $^{15-18}$ or fixed, denatured tissue samples. ${ }^{19-21}$ Our work presents phase contrast images of freshly dissected mastectomy samples obtained with a conventional, low-brilliance $\mathrm{x}$-ray tube evaluated on the basis of 2 blinded, prospective independent reader studies. We present a first comprehensive statistical evaluation of the imaging capabilities of this emerging $\mathrm{x}$-ray technique compared with digital absorption mammography.

\section{MATERIALS AND METHODS}

\section{Study Design}

Two prospective reader studies were designed, involving mastectomy specimens of 33 patients and 6 international breast radiologists 
working in certified European breast units and screening programs. Mammograms of the freshly resected breasts were obtained with absorption-based clinical digital mammography (Mx-clin). Furthermore, absorption-based digital mammography (Mx-exp) and phase contrast-enhanced mammography (mammoDPC) were acquired simultaneously on an experimental setup as described in the study of Stampanoni et al. ${ }^{14}$

The first study (study 1) directly compared images obtained with our experimental setup in absorption (Mx-exp) and mammoDPC using the same dose (reported to be $26.2 \mathrm{mSv}$ in the study of Stampanoni et $\mathrm{al}^{14}$ ). This allows the a priori removal of instrument-specific characteristics and yields system-unbiased images. Craniocaudal (CC) and anteroposterior (AP) views were evaluated. Mediolateral (ML) and mediolateral oblique (MLO) views were not considered because they are almost impossible to obtain with the resected breast. Dedicated questionnaires on the general quality of the image, sharpness and delineation of the lesions, microcalcifications, skin involvement, artifacts, and general clinically relevant information were prepared. Study 1 included 9 comparative questions assessing the relative strength of phase contrast versus absorption mammograms on a 5-point Likert scale ("highly superior," "superior," “equivalent," "inferior," "highly inferior") with respect to the previously mentioned criteria. All readers were equally instructed by the same person and independently evaluated the images presented on screen after the questionnaire.

The second study (study 2) investigated 3 types of images for all 33 mastectomy samples: Mx-clin, Mx-exp, and mammoDPC. All types of mammograms were evaluated independently as randomized blinded images by the readers. Findings of images obtained with Mxclin were compared with those of Mx-exp and mammoDPC. In study 2 , only CC views are included because they correspond to the imaging plane that is regularly acquired in clinical practice. Also, for study 2, dedicated questionnaires, including 12 questions evaluating different aspects as returned by clinical, experimental, and phase contrast mammography on a multiple-point Likert scale, were prepared. All readers, again, were equally instructed by the same person and independently evaluated the randomized images presented on screen after the questionnaire. Because of experimental limitations, the dose for Mx-exp and mammoDPC was approximately 25 times larger than the dose for $\mathrm{Mx}$-clin, as explained in the study of Stampanoni et al. ${ }^{14}$ This issue does not affect study 1 (comparison of Mx-exp with mammoDPC with the same dose) and is marginally relevant for study 2. The higher dose reported for mammoDPC is mainly caused by the use of an unfiltered source, causing low-energy photons to be absorbed in the sample and interferometer but, finally, not contributing to image formation.

Both reader studies were blinded because all readers independently evaluated all mammograms taken with the 3 methods in a randomized order. In reader study 1 in particular, where Mx-exp was directly compared with mammoDPC, the randomization order also included 2 view planes (CC and AP).

Histopathological findings were available for all mastectomy samples included in the study. Identification and localization of histopathologically proven ductal carcinoma in situ (DCIS) were used to correlate image readings (positive and negative findings). All routine hematoxylin-eosin stained slides were reviewed by a gynecopathologist.

\section{Patients Included and Sample Preparation}

The local institution's ethical review board approved this study, and written informed consent was obtained from all patients. Men or women older than 18 years who presented at the department of gynecology between November 2010 and April 2012 with histopathologically confirmed unifocal, multifocal, or multicentric invasive ductal or lobular breast cancer, with and without additional DCIS, requiring modified radical mastectomy, and willing to participate in the study, were considered eligible. Patients who qualified for breast-conserving operative procedures, skin sparing, or subcutaneous mastectomy were excluded. The non-fixed tissue samples were investigated immediately after mastectomy. Breast density values according to American College of Radiology (ACR) criteria, ${ }^{22}$ available from in vivo mammography performed on a MAMMOMAT Inspiration system (Siemens Healthcare, Erlangen, Germany) at the department of radiology of the Kantonsspital Baden 1 to 30 days before surgery, were compared with the ex vivo values provided by the study readers. Table 1 summarizes the histopathological findings and breast density values of the patients (one 55-year-old man and 32 women with a mean age of 67 years [range, 43-93 years]) including the type of invasive carcinoma, histological grade, histopathological presence of additional DCIS, tumor centricity by histopathology, and microscopic skin infiltration.

The surgical procedure involved a modified radical mastectomy followed by a sentinel lymph node dissection and/or a complete axillary lymph node clearance, when clinically indicated. The removed breast specimen was marked with small metal clips to unequivocally identify the 3 -dimensional orientation. The breast tissue samples were then mounted immediately after resection into a dedicated, cooled breast tissue holder, and within 30 to 90 minutes after the resection, Mx-clin as well as simultaneous Mx-exp and mammoDPC imaging were performed. The breast tissue holder was designed to mimic the correct orientation and adequate compression of the tissue compared with the in vivo situation. ${ }^{14}$ Absorption mammography of the breast sample (Mx-clin) was performed with a fully digital system (MAMMOMAT Inspiration; Siemens Healthcare, Erlangen, Germany). Formalin fixation and standard histopathological examination were initiated after the mammoDPC acquisition.

TABLE 1. Histopathological and Radiological Findings of the 33 Cases Included in the Study

\section{Grading,}

According to

the TNM

Tumor Centricity

Classification

\begin{tabular}{|c|c|c|c|c|c|c|c|c|c|c|c|}
\hline & \multirow[b]{2}{*}{ No. Cases } & & & \multirow[b]{2}{*}{ ACR I } & \multirow[b]{2}{*}{ ACR II } & \multirow[b]{2}{*}{ ACR III } & \multirow[b]{2}{*}{ ACR IV } \\
\hline & & Unifocal & Multifocal & Multicentric & G1 & G2 & G3 & & & & \\
\hline Invasive ductal carcinoma & 28 & 20 & 2 & 6 & 0 & 9 & 19 & 6 & 4 & 9 & 9 \\
\hline Invasive lobular carcinoma & 5 & 0 & 0 & 5 & 0 & 5 & 0 & 0 & 0 & 4 & 1 \\
\hline Thereof DCIS & 22 & 14 & 1 & 7 & 0 & 9 & 13 & 3 & 3 & 6 & 10 \\
\hline Thereof skin infiltration & 11 & 5 & 2 & 4 & 0 & 4 & 7 & 6 & 1 & 2 & 2 \\
\hline
\end{tabular}

ACR indicates American College of Radiology; DCIS, ductal carcinoma in situ. 


\section{Grating Interferometry and mammoDPC Measurement}

A Talbot-Lau ${ }^{23}$ grating interferometer with a standard $\mathrm{x}$-ray tube and a commercial flat panel detector (pixel size, $50 \times 50 \mu \mathrm{m}$ ) was set up at the Paul Scherrer Institute in Villigen, Switzerland. We used Seifert ID 3000 x-ray generator, an unfiltered tungsten line focus tube (operated at 40 kilovolt [peak] with a mean energy of $28 \mathrm{keV}$ and a current of $25 \mathrm{~mA}$ ), and a 3 grating interferometer (with periods of $p_{0}=14 \mu \mathrm{m}, p_{1}=3.5 \mu \mathrm{m}$, and $p_{2}=2.0 \mu \mathrm{m}$, respectively). The source-to-detector distance was $1.6 \mathrm{~m}$. Additional details on the experimental system are given in the study of Stampanoni et al. ${ }^{14}$ Absorption, phase, and scattering signals were recorded simultaneously using a phase-stepping approach, where the absorption grating is translated perpendicular to the grating direction by a fractional distance of the grating period, and a Fourier analysis of the resulting intensity curve returned the previously mentioned signals. ${ }^{24}$ After the operation, Mx-clin was performed in the clinic, whereas Mx-exp and mammoDPC were performed within 30 to 90 minutes after transport (15-km distance) at the Paul Scherrer Institute in Villigen, Switzerland. The field of view of the Mx-exp and mammoDPC images is limited to $5 \times 5 \mathrm{~cm}^{2}$, thus requiring multiple snapshots to be tiled together to cover the size of the resected breast.

\section{Imaging Protocol and Image Processing}

The raw Mx-exp images were processed with the Philips UNIQUE algorithm, ${ }^{25}$ which is used in commercial mammography systems. This ensures that the quality of the processed images corresponds to the clinical standard. During the mammoDPC data acquisition, one of the interferometer gratings is moved perpendicular to the grating lines within at least 1 gratings period (phase-stepping approach). For each pixel on the detector, a quasi-sine intensity curve is recorded. The absorption, differential phase, and scattering signals are obtained by a retrieving algorithm ${ }^{24,26}$ operating on phase-stepping curves with and without the object.

The retrieved signals have been effectively fused into a single and more informative image. The fusion procedure involves merging the high frequencies of the differential phase signal into the absorption image by a dedicated algorithm. ${ }^{28}$ The inclusion of the scattering image is achieved by fusing it into the merged image by adaptive, multilevel thresholding. ${ }^{28}$ It is based on a "decompose-process and fusereconstruct" approach. The absorption, differential phase, and scattering signals are first decomposed into multiple levels and bands, which represent image information at different scales and frequencies, with the help of wavelets decomposition. Furthermore, an intraband processing first enhances the local signal-to-noise ratio; then, the interband processing weights each band by considering its characteristics and relative contributions. Finally, the resulting fusion image shows a similar appearance to that of a conventional mammogram but contains significantly enhanced features' details.

\section{Statistical Analysis}

The statistical analysis was done by the statistical consulting service of the Institute of Social and Preventive Medicine, University of Zurich, Zurich, Switzerland. The Wilcoxon signed rank test was used for the qualitative analysis of subjective preference score in both reader studies. IBM Statistical Package for the Social Sciences Statistics 20 (SPSS Inc, Chicago, IL) was used for the descriptive and pairwise comparisons (Wilcoxon signed rank test) of image types. Stata 11.2 (StataCorp, Lakeway Drive, TX) was used for the computation of $\kappa$ values to evaluate interreader variability. For all statistical analyses, a 2 -tailed $P<0.05$ was considered to indicate a statistically significant difference. For reader study 1 , a Bonferroni correction was made to consider the 9 comparisons per view; therefore, $P \leq 0.05 / 9$ approximately equal to 0.005 was considered significant. For reader study 2 , a total of 2 comparisons per question were considered, resulting in $P \leq 0.05 / 2$ equal to 0.025 for significance. Interobserver agreement between the readers was calculated by weighted $\kappa$ statistics. The $\kappa$ values in the ranges 0.00 to $0.20,0.21$ to $0.40,0.41$ to $0.60,0.61$ to 0.80 , and 0.81 to 1.00 were considered to represent slight agreement, fair agreement, moderate agreement, substantial agreement, and almost perfect agreement, respectively.

\section{RESULTS}

\section{Reader Study 1}

The results of the statistical analysis revealed the general quality of the mammoDPC images to be significantly superior than that of the Mx-exp based images $(P<0.001$; median of 2 with an interquartile range [IQR] of 2-3, with 2 being "superior" and 3 being "equivalent"). Furthermore, the sharpness and lesion delineation as well as the general visibility of calcifications were rated as significantly more assessable on mammoDPC than on Mx-exp $(P<0.001$; median of 2 with an IQR of 2-3, with 2 being "superior" and 3 being "equivalent"). MammoDPC images resulted in a sharper delineation of anatomic components of the specimens (surface structures) when evaluating the periphery of the images $(P<0.001$; median of 2 with an IQR of $2-2$, with 2 being "superior"), with $75 \%$ of the readings indicating that mammoDPC was better than Mx-exp. Table 2 summarizes the results.

\section{Reader Study 2}

Spiculations could be significantly better identified in mammoDPC images compared with Mx-clin $(P<0.015)$, whereas Mx-exp images were shown to be directly comparable with the Mx-clin mammograms $(P<0.076)$ (Figs. 1 and 2C, D). Blood vessel visibility was lower in the mammoDPC and Mx-exp images, when compared with the Mx-clin mammograms (Table 3).

\section{Microcalcifications}

The presence of clusters of suspicious microcalcifications was similarly observed in mammoDPC with respect to Mx-exp. No statistically significant difference $(P=0.2)$ was observed (Table 3$)$. On the other hand, significantly superior findings of calcification sharpness (Table 2) were seen in mammoDPC compared with Mx-exp $(P<0.001$; median of 2 with an IQR of $2-3$, with 2 being "superior" and 3 being "equivalent") (Figs. 1A, B and 2A, B).

TABLE 2. Statistical Outcome Showing the Criteria Under Which mammoDPC Is Superior to Absorption-Based Mammography

\begin{tabular}{lcc}
\hline Evaluated Criteria (mammoDPC Is Superior) & $\boldsymbol{P}^{*}$ & IQR \\
\hline General quality of image & $<0.001$ & $2-3 \dagger$ \\
Sharpness and lesion delineation & $<0.001$ & $2-3$ \\
Delineation of surface structures & $<0.001$ & $2-3$ \\
Sharpness of microcalcifications & $<0.001$ & $2-2$ \\
General visibility of microcalcifications & $<0.001$ & $2-3$ \\
Potentially clinically relevant information & $<0.001$ & $4-5$ † \\
Identification of spiculations & $<0.015 \S$ & \\
\hline
\end{tabular}

$* P<0.005$ is considered to be significant with Bonferroni correction.

$\dagger 2$ being "superior" and 3 being "equivalent" quality.

$\$ 4$ being " $11 \%$ to $20 \%$ superior" and 5 being " $1 \%$ to $10 \%$ superior."

$\S$ This criterion was evaluated in study 2. $P<0.025$ is significant with the Bonferroni correction.

IQR indicates interquartile range; mammoDPC, phase contrast-enhanced mammography. 

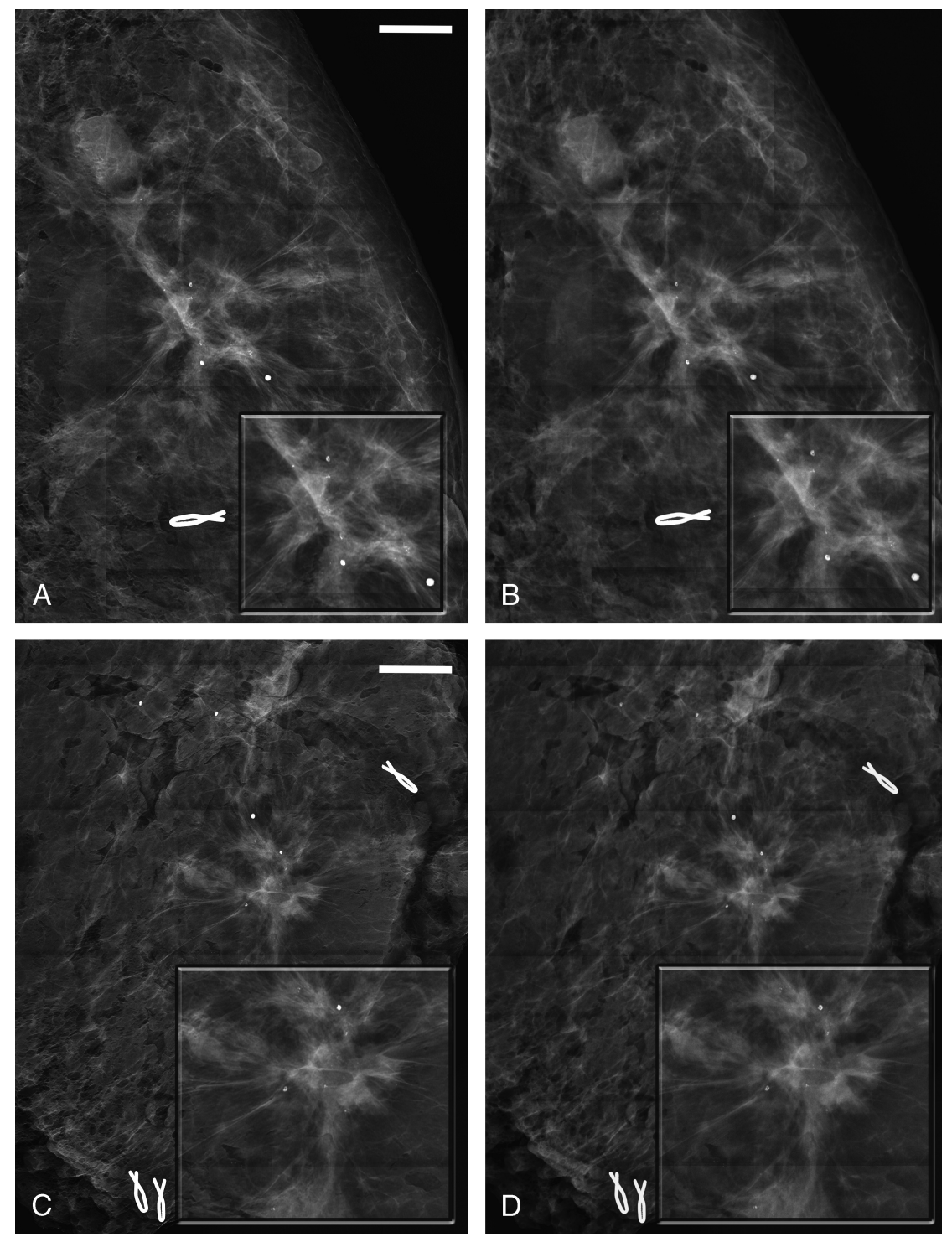

FIGURE 1. $A$ and $B$, Craniocaudal view of native breast tissue sample after mastectomy. $C$ and $D$, Anteroposterior view of the same breast sample. A and C were obtained with mammoDPC; $B$ and D, with Mx-exp. A sharpness increase in mammoDPC was observed for microcalcifications $(A)$ and for spiculations $(C)$. Visibility and delineation of structural details were improved in mammoDPC $(A, C)$ compared with $M x-\exp (B, D)$. The scale bar in $A$ and $C$ is $2 \mathrm{~cm}$.

\section{Ductal Carcinoma In Situ}

We analyzed the ability of Mx-clin, Mx-exp, and mammoDPC imaging to detect microcalcifications interpreted by the readers as suggestive of DCIS. Although the results were not statistically significant (Table 3), we observed that mammoDPC returned more true findings (64\%, positives and negatives) than did Mx-exp (56\%) and Mx-clin (59\%) when related to the criterion standard provided by the histopathological examination. It must be stated, however, that we obtained a considerable amount of false negatives (36\%, 37\%, and $29 \%$ for Mx-clin, Mx-exp, and mammoDPC, respectively) for this diagnostic question, with a moderate level of interreader agreement $(\kappa=0.400)$. Because of the inclusion criteria of the study, there was only 1 patient with no radiological signs of invasive carcinoma. This patient had a very small invasive carcinoma (pT1a), surrounded by an extended DCIS. All other patients showed histopathological findings consistent with an invasive carcinoma of $\mathrm{pT} 1 \mathrm{~b}$ or higher. This was successfully indicated by all 3 methods, with $91 \%$ of true positives for mammoDPC compared with $87 \%$ for both Mx-exp and Mx-clin. This difference was not statistically significant and the level of interreader agreement was moderate $(\kappa=0.546)$.

\section{Artifacts}

Minor or no imaging artifacts (like abnormal edge enhancement) were detected in most of the readings $(94 \%, 97 \%, 82 \%$ for Mx-clin, Mx-exp, and mammoDPC, respectively). More generally, mammoDPC generated significantly stronger artifacts than did Mx-clin $(P<0.001)$, whereas it was comparable with Mx-exp $(P=0.047$ with a threshold of 0.025 for significance, according to Bonferroni). The presence of new types of artifacts, generated by the intrinsic physics of the novel signals and the experimental arrangement, led to a slight change in interpretation, but it did not affect the diagnostic content. Among the pictures showing artifacts (disregarding air bubbles and non-tissue related 

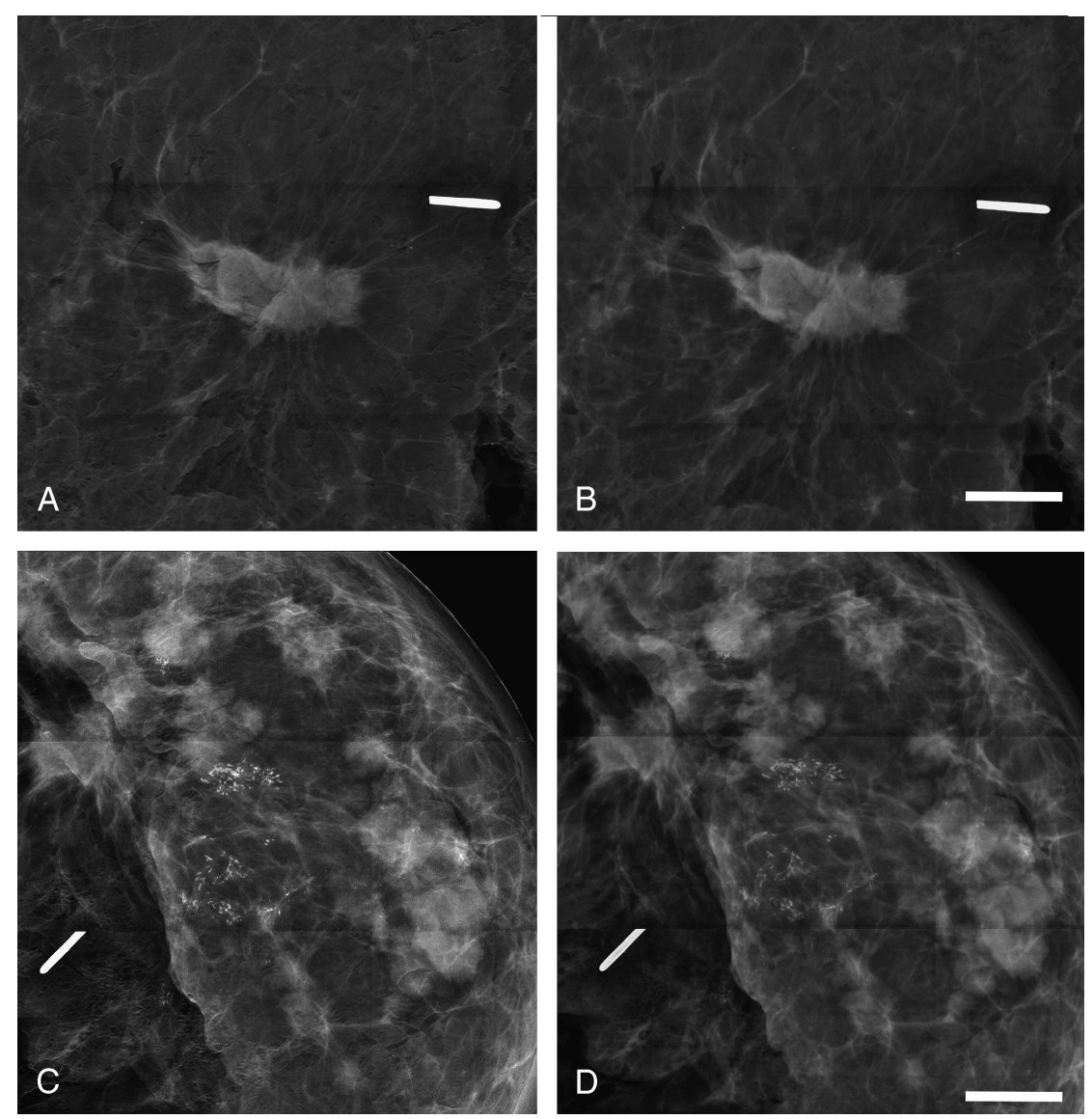

FIGURE 2. Anteroposterior $(A-B)$ and $C C(C-D)$ views of a native breast tissue sample from 2 different patients after mastectomy. $A$ and $C$ were obtained with mammoDPC; $B$ and $D$, with Mx-exp. Looking at the margin between lesions and the surrounding tissue, one can observe a sharper delineation of surface structures in mammoDPC (A) compared with Mx-exp (B), particularly at the periphery of the images. Furthermore, mammoDPC showed a generally superior visibility and sharpness of the microcalcifications (C vs D). The scale bar is $2 \mathrm{~cm}$.

structures due to the experimental arrangement), $75 \%$ of the readings classified those artifacts in mammoDPC as significantly stronger than those in Mx-clin $(P<0.001$; an IQR of 2-2, with 2 being "stronger" and 3 being "equivalent"). In reader study 2 , air bubbles or other non-tissue related structures were identified for all mammography techniques by all readers. Specifically, with Mx-clin, air bubbles were visible in $88 \%$ of the readings, whereas Mx-exp and mammoDPC showed these artifacts in $95 \%$ and $99 \%$ of the readings, respectively. This difference was not statistically significant.

\section{Skin Infiltration}

Approximately one third of the tumors showed histopathologically confirmed skin infiltration. No significant differences were found in the abilities of the 3 approaches to detect signs of skin infiltration (skin retraction and/or skin thickening; Table 3). However, the number of cases was insufficient for a sound statistical analysis. The level of interreader agreement for this evaluation was fair $(\kappa=0.236)$.

\section{Potentially Clinically Relevant Information}

One of the most challenging questions was related to the amount of potentially clinically relevant information provided by the novel method to the readers according to their individual judgments weighted by their expertise. The readers compared all pairs of images and indicated an amount of potentially superior or inferior clinically relevant information of Mx-exp compared with mammoDPC. Excluding equivalent findings
$(72 \%)$ resulted in a significant superiority of the mammoDPC technique with respect to $\mathrm{Mx}$-exp $(P<0.001$; median of 5 with an IQR of $4-5$, with 5 being " $1 \%$ to $10 \%$ superior" and 4 being " $11 \%$ to $20 \%$ superior").

The ACR breast density values evaluated among all 3 imaging protocols were not significantly different from those of in vivo mammography (considered as the criterion standard), demonstrating that, as

TABLE 3. Statistical Outcome Showing the Criteria Under Which mammoDPC Is Inferior/Equivalent to Absorption-Based Mammography

\begin{tabular}{lc}
\hline Evaluated Criteria (mammoDPC Is Inferior) & $\boldsymbol{P}^{*}$ \\
\hline Lower blood vessel visibility & $<0.018$ \\
More artifacts & $<0.001$ \\
Signs of skin infiltration & $\mathrm{N} / \mathrm{A} \dagger$ \\
Clusters suggestive of microcalcifications & $\mathrm{N} / \mathrm{A}$ \\
Signs of the presence of DCIS & N/A \\
\hline
\end{tabular}

$* P<0.025$ is considered to be significant with the Bonferroni correction. $\dagger$ No significance was found.

DCIS indicates ductal carcinoma in situ; mammoDPC, phase contrastenhanced mammography. 
expected, Mx-clin as well as Mx-exp and mammoDPC did not affect breast density scoring. The level of interreader agreement for this evaluation was moderate $(\kappa=0.480)$.

\section{DISCUSSION}

The mammoDPC images were preferred over the Mx-clin and Mx-exp mammograms with respect to potential clinical relevance, according to the general quality of the mammoDPC images, sharpness and lesion delineation as well as the general visibility of calcifications, sharper delineation of anatomic components of the specimen, and spiculation visibility. This preference was statistically significant when the number of equivalent findings was excluded from the analysis. This result can be explained by the fact that the study was carried out exclusively with mastectomy samples, that is, where a malignant process was previously identified.

The identification of DCIS in the mammograms proved to be difficult for all readers. Because no patient with only DCIS was included in the study, the diagnostic challenge was to identify DCIS within or surrounding an invasive carcinoma.

Blood vessel visibility was found to be lower in mammoDPC and Mx-exp than in Mx-clin $(P=0.017$ for mammoDPC vs Mx-clin with a threshold of 0.025 for significance, according to Bonferroni; Table 3). This evidence may also be a consequence of the study design. The Mx-clin mammogram was taken only a few minutes after the resection, when blood was still in the vessels and could contribute to the contrast. The Mx-exp and mammoDPC were performed within 30 to 90 minutes, but during this time, the remaining blood redistributed within the sample. As the sample is mounted into the dedicated breast holder with a compression that simulates clinical standards, a loss of blood occurs and affects the contrast in the subsequent investigation. This interpretation is supported by the finding that mammoDPC and Mx-exp showed comparable readings with respect to blood vessel visibility $(P=0.25)$.

The results of the evaluation of signs for skin infiltration did not reveal any significant differences between the 3 methods. Therefore,
mammoDPC does not affect, either positively or negatively, the ability to identify signs of skin infiltration (Table 3).

Existing scar tissue due to previous surgical interventions consists of a different extracellular matrix structure ${ }^{29,30}$ resulting from a change in the quantity and type of collagens and noncollagenous components, when compared with regular breast tissue, and is well detectable by mammoDPC, as shown in Figure 3. This is caused by the high sensitivity of the technique to the scattering power of the investigated sample. Similarly, nontissue material present in the breast such as stitches that usually consist of strongly scattering textile wires cannot be detected by Mx-clin but is strongly enhanced by mammoDPC.

\section{Study Limitations}

Because of the inclusion criteria of our study design, 33 breast samples of all patients screened during the allocated time frame have been included. Despite being blinded, the readers quickly learned how to differentiate between $\mathrm{Mx}$-clin/Mx-exp and mammoDPC because of the striking sharpness increase of the edges within the mammoDPC images. This was unavoidable because mammoDPC is expected to increase sharpness in general. The "tiling" artifact due to the limited field of view observed in both the Mx-exp and mammoDPC images was not reported to be a disturbing factor in the reading process. Nevertheless, an ongoing study (unpublished) demonstrates that gratings can be successfully stitched to cover a much larger field of view, eliminating every tiling artifact in future experiments. Furthermore, the readers may be biased by the fact that they had to read images of mastectomy samples because, as a consequence of the study design, no resected whole-breast sample without invasive carcinoma was included. The strong enhancement of fine details, due to the boost in high-frequency content by the differential phase contrast, ${ }^{28}$ was sometimes ranked as an artifact. This may have arisen because the radiologists involved in the study were trained to interpret absorption-based mammograms. We are aware that radiologists interpreting mammograms must be particularly attuned to the detection of the presence of microcalcifications, which can also be considered high-frequency image data. However,
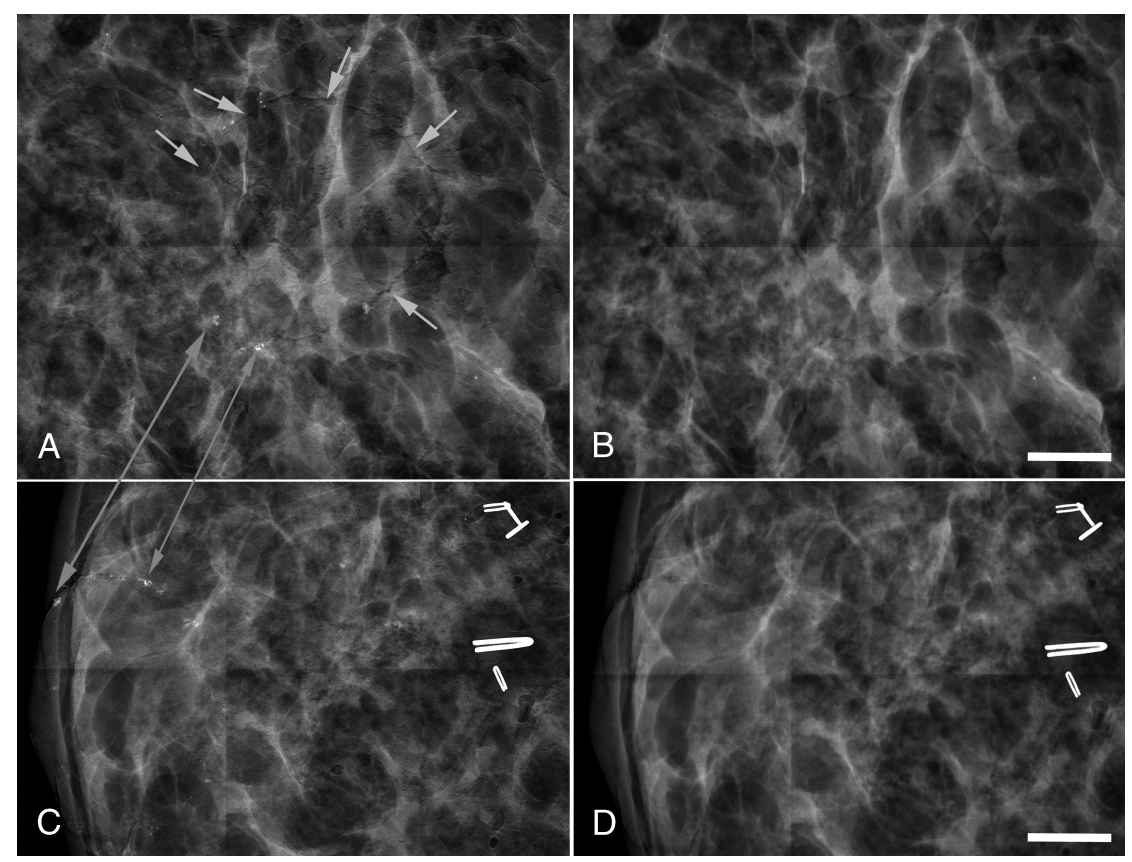

FIGURE 3. $A$ and $B, C$ raniocaudal view of native breast tissue after mastectomy. $C$ and $D$, Anteroposterior view of the same breast. The patient underwent a breast-conserving intervention before mastectomy. A and $\mathrm{C}$ were obtained with mammoDPC; $B$ and D, with Mx-exp. In the mammoDPC image, signals from scars (arrowheads) and internal stitches (double-headed arrows) are clearly visible. In the Mx-exp images, the stitches are invisible, whereas the scars are barely seen. The scale bar is $2 \mathrm{~cm}$. 
microcalcifications are normally identified as bright, localized spots, whereas the previously mentioned frequency boosting happens all over the image. Also, there was no presence of microcalcifications but only subtle tissue changes. The mean glandular dose for a $4.5 \mathrm{~cm}$ thick breast sample was measured with a PTW-UNIDOS instrument to be $26.2 \mathrm{mSv}$ for both mammoDPC and Mx-exp, as described in the study of Stampanoni et al. ${ }^{14}$ The high delivered dose $(25$ times higher as recommended in the European guidelines ${ }^{31}$ ) is mainly caused by the nonoptimized design of the experimental instrument. Because of its low photon efficiency, the delivered dose is deliberately tuned higher to be able to generate Mx-exp images with similar image quality to that of Mx-clin images for fair comparison. With an optimized design of each component, as described in the study of Stampanoni et al, ${ }^{14}$ the dose could be reduced by more than 1 order of magnitude while maintaining the same image quality.

With this study, we have demonstrated, for the first time, that phase contrast-enhanced mammograms of mastectomy samples obtained using a conventional x-ray source have significantly higher diagnostic content compared with that of classically digital absorption-based images for the evaluated criteria as summarized in Table 2. Differential phase contrast mammography may have the potential to advance as a new diagnostic tool and eventually outperform recently introduced approaches, for instance, the use of monochromatic x-rays for digital mammography. ${ }^{32}$ So far, no other imaging technology is capable of exploiting x-ray differential phase and scattering signals to significantly improve soft tissue imaging. These results are promising toward the use of phase contrast grating interferometry in vivo in a clinical setting. On the basis of our experience, ${ }^{14}$ we expect this technology to be further developed and integrated into an optimized prototype performing at dose depositions lower or (at worst) comparable with present, approved instruments. The complementary information provided by phase and scattering-enhanced mammograms delivers images of generally superior quality. As a consequence, this technique has the potential to improve radiological breast diagnostics.

\section{ACKNOWLEDGMENTS}

The authors thank E. Schneider and A. Peters, radiology technicians at the Department of Radiology, Kantonsspital Baden, Baden, Switzerland, for their help in running Mx-clin specimen mammograms after mastectomy. Moreover, the authors thank I. Schulze-Wenck from Philips Healthcare, Hamburg, Germany, for her support with the instruction of the readers. Also, the authors thank the breast radiologists who participated as readers: Prof Dr R. A. Kubik-Huch, Prof Dr I. Schreer, Prof Dr A. Mundinger, Prof Dr S. Montemezzi, Dr B. FreiwaldChilla, and Dr S. Saravanja. All readers are experts in breast diagnostics with many years of experience. Prof Dr R. Kubik-Huch critically revised the manuscript, after the blinded reader study and statistical analysis were completed. The authors thank Prof Dr Burkhardt Seifert, Division of Biostatistics, Institute of Social and Preventive Medicine, University of Zurich, Zurich, Switzerland, for his support in the statistical analysis.

\section{REFERENCES}

1. Lewis RA. Medical phase contrast x-ray imaging: current status and future prospects. Phys Med Biol. 2004;49:3573-3583.

2. Pfeiffer F, Bech M, Bunk O, et al. Hard-x-ray dark-field imaging using a grating interferometer. Nat Mater. 2008;7:134-137.

3. Donnelly EF, Lewis KG, Wolske KM, et al. Characterization of the phasecontrast radiography edge-enhancement effect in a cabinet x-ray system. Phys Med Biol. 2006;51:21-30.
4. Margaritondo G, Meuli R. Synchrotron radiation in radiology: novel x-ray sources. Eur Radiol. 2003;13:2633-2641.

5. Ando M, Hashimoto E, Hashizume H, et al. Clinical step onward with X-ray dark-field imaging and perspective view of medical applications of synchrotron radiation in Japan. Nucl Instrum Meth A. 2004;548:1-16.

6. Lewin JM, Hendrick RE, D'Orsi CJ, et al. Comparison of full-field digita mammography with screen-film mammography for cancer detection: results of 4,945 paired examinations. Radiology. 2001;218:873-880.

7. Pisano ED, Gatsonis C, Hendrick E, et al. Diagnostic performance of digital versus film mammography for breast-cancer screening. $N$ Engl $J$ Med. 2005; 353:1773-1783.

8. Barlow WE, Lehman CD, Zheng YY, et al. Performance of diagnostic mammography for women with signs or symptoms of breast cancer. $J$ Natl Cancer I. 2002;94:1151-1159.

9. Cole E, Pisano ED, Brown M, et al. Diagnostic accuracy of Fischer SenoScan digital mammography versus screen-film mammography in a diagnostic mammography population. Acad Radiol. 2004;11:879-886.

10. Nelson HD, Tyne K, Naik A, et al. Screening for breast cancer: an update for the U.S. Preventive Services Task Force. Ann Intern Med. 2009;151:727-737.

11. Kuhl CK, Kuhn W, Schild H. Current status of breast MR imaging. Part 2. Clinical applications. Radiology. 2007;244:672-691.

12. Haas BM, Kalra V, Geisel J, et al. Management of women at high risk for breast cancer: new imaging beyond mammography. Breast. 2005;14:480-486.

13. Poplack SP, Tosteson TD, Kogel CA, et al. Digital breast tomosynthesis: initial experience in 98 women with abnormal digital screening mammography. AJR Am J Roentgenol. 2007;189:616-623.

14. Stampanoni M, Wang $Z$, Thuering $T$, et al. The first analysis and clinical evaluation of native breast tissue using differential phase-contrast mammography. Invest Radiol. 2011;46:801-806.

15. Fiedler S, Bravin A, Keyrilainen J, et al. Imaging lobular breast carcinoma: comparison of synchrotron radiation DEI-CT technique with clinical CT, mammography and histology. Phys Med Biol. 2004;49:175-188.

16. Dreossi D, Abrami A, Arfelli F, et al. The mammography project at the SYRMEP beamline. Eur J Radiol. 2008;68:58-62.

17. Keyrilainen J, Fernandez M, Fiedler S, et al. Visualisation of calcifications and thin collagen strands in human breast tumour specimens by the diffractionenhanced imaging technique: a comparison with conventional mammography and histology. Eur J Radiol. 2005;53:226-237.

18. Coan P, Bamberg F, Diemoz PC, et al. Characterization of osteoarthritic and normal human patella cartilage by computed tomography $\mathrm{X}$-ray phase-contrast imaging: a feasibility study. Invest Radiol. 2010;45:437-444.

19. Williams IM, Siu KKW, Gan R, et al. Towards the clinical application of x-ray phase contrast imaging. Eur J Radiol. 2008;68(suppl 3):73.

20. Tanaka T, Honda C, Matsuo S, et al. The first trial of phase contrast imaging for digital full-field mammography using a practical molybdenum $\mathrm{x}$-ray tube. Invest Radiol. 2005;40:385-396.

21. Donath T, Pfeiffer F, Bunk O, et al. Toward clinical x-ray phase-contrast CT. Demonstration of enhanced soft-tissue contrast in human specimen. Invest Radiol. 2010;45:445-452.

22. ACR. ACR BI-RADS $®$ - Mammography. ACR Breast Imaging Reporting and Data System, Breast Imaging Atlas. 2003.

23. Pfeiffer F, Weitkamp T, Bunk O, et al. Phase retrieval and differential phasecontrast imaging with low-brilliance x-ray sources. Nat Phys. 2006;2:258-261.

24. Weitkamp T, Diaz A, David C, et al. X-ray phase imaging with a grating interferometer. Opt Express. 2005;13:6296-6304.

25. Stahl M, Aach T, Dippel S. Digital radiography enhancement by nonlinear multiscale processing. Med Phys. 2000;27:56-65.

26. Momose A, Kawamoto S, Koyama I, et al. Demonstration of X-Ray Talbot interferometry. Jpn J Appl Phys. 2003;42:L866.

27. Roessl E, Koehler T, van Stevendaal U, et al. Image fusion algorithm for differential phase contrast imaging. Proc SPIE: Phys Med Imaging. 2012; 8313:831354.

28. Wang Z, Clavijo CA, Roessl E, et al. Image fusion scheme for differential phase contrast mammography. J Instrum. 2013;8:C07011.

29. Eckes B, Nischt R, Krieg T. Cell-matrix interactions in dermal repair and scarring. Fibrogenesis Tissue Repair. 2010;3:1-11.

30. Krieg T, Hein R, Hatamochi A, et al. Molecular and clinical aspects of connective tissue. Eur J Clin Invest. 2012;18:105-217.

31. Perry N, Broeders M, de Wolf C, et al. European guidelines for quality assurance in breast cancer screening and diagnosis. Fourth edition-summary document. Ann Oncol. 2008;19:614-622.

32. Yoon KH, Kwon YM, Choi BJ, et al. Monochromatic X-rays for low-dose digital mammography: preliminary results. Invest Radiol. 2012;47:683-687. 